\title{
LEVI-CIVITA ON MECHANICS
}

Lezioni di Meccanica Razionale. By Tullio Levi-Civita and Ugo Amaldi. Vol. 1, 2d Edition, Vol. 2, parts 1 and 2, 1st Edition. Bologna, Nicola Zanichelli, 1926-30. Pp. 807, 526, 684.

These books take their place along with such treatises as those of Routh, Appell, Budde, Resal, and Lecornu, both in quality and extensiveness. Together they comprise a good many pages. Nevertheless they are limited to the statics and dynamics of particles and rigid bodies. They do not digress extensively into special fields; no matter is set off in small type to invite omission; the principles and methods of mechanics are developed from the more elementary to the more advanced; the applications given are those of which the importance or interest is readily seen. There is no effort at terseness or brevity of expression. The authors are not only able, but are willing to lay the matter fully before the reader. The work is not merely a sequence of mathematical formulas pieced together with a minimum of sentences, whose meaning may, or may not, be fully comprehended by the reader. Equations in the last analysis must be the supporting elements of any work on dynamics. But once the equations have been definitely discovered and accepted, little skill may be required to piece them together in something like a logical sequence. But a person who does this is rather a compiler than an author. The present volumes are a real narrative, interesting and readable, worthy of occupying a high place in a literature already distinguished.

The work opens with a chapter on vector analysis. No work on mechanics can be regarded as fully adequate that shuns the use of vectors where they undoubtedly contribute to clarity and simplicity. In the present case they are used judiciously, appearing here and there throughout the entire work. The introductory chapter develops the subject distinctly as a tool, and one is not led astray through a mere attachment for the subject.

The next five chapters, comprising a total of nearly 250 pages, are devoted to kinematics. They state conspicuously at the start that mechanics presupposes geometry, and adds to this, as its own peculiar fundamental concept the notion of time. Attention is called to the fact that within recent years a great change has taken place in our ideas of time, which formerly was taken as primitive and dismissed at that. Only a page is devoted to the newer relativity aspect of time, but the cardinal idea is set forth, and enough is said perhaps to arouse such interest in the reader as will lead him to consult fuller and special treatments. The plan of developing at the beginning all matters of a kinematical nature, whether they pertain to a particle or a rigid body, has of course a logical advantage. But it has the disadvantage of delaying the real subject, that of dynamics. It is not until more than 300 pages have been read that one encounters the idea of a force; differential equations of motion first occur on page 358. Some of the kinematical ideas that relate to a rigid body are not simple. It is likely that even an attentive reader will find it necessary, when he finally reaches the dynamics of the rigid body, to study again such a question as that of the angles of Euler, quite as if it were all new material. 
One is pleased to find the consideration of dynamics proper starting with a chapter bearing such a title as "The Concepts and Fundamental Postulates of Mechanics." One reads with attentive interest the way in which the idea of force is developed, and following it, that of mass. A careful study of the forty pages of the chapter should answer some of the questions that often trouble the careful student.

Volume II, part 1, treats the more customary problems of particles and systems. The elements of celestial mechanics receive a fairly satisfactory chapter of 50 pages, including a few words on perturbations. The equations of Lagrange are introduced, and one encounters the symbols of Christoffel. The concluding chapter, of considerable length, is devoted to stability and vibrations.

The second part of volume II opens with the systematic study of the rigid body, and the matter is carried through about 300 pages. Then comes the portion of the work that one looks through with particular interest - the discussion of the more advanced aspects of dynamics. In such questions as the derivation and discussion of the canonical equations, the avoidance of an extreme terseness is particularly welcome. The transformation theory of dynamics, of which there are not many clear and satisfactory expositions, can be read here with understanding. The Hamilton-Jacobi theory is well set forth, and the splendid astronomical application is carried farther than in most works. But the perturbation theory of astronomy is such an extensive subject, so full of peculiar difficulties, that one can doubt if mere hinting at it can give the reader any adequate conception of it at all. The work closes with a chapter of nearly a hundred pages on impulsive motion.

A very notable feature of the work is the biographical notes. Whenever any mathematician is mentioned, and care is taken to assign the more important parts of the subject, a note occurs that sets forth the main facts about the life and work of the man. These notes sometimes glow with a warmth of appreciation that cannot fail to impress the reader.

Each chapter is followed by a list of exercises. They are of a vary substantial character; at times they constitute important supplements to the text. In one instance the exercises cover 35 pages, in another 32 pages. In all, the exercises comprise 300 pages out of a total of 2000 The authors have not only produced a text proper of unusual clearness; they have given an abundance of material for the exercise and development of the reader's own ability.

K. P. Williams 